\title{
Introducing «dermatogeriatrics»: the need for geriatric dermatology as a subspecialty
}

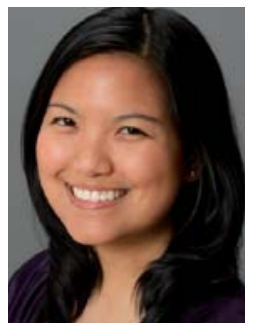

Jillian W. Wong

Clinical Research Investigator at the University of California, San Francisco, Psoriasis and Skin Treatment Center; Medical Student at the University of Utah, School of Medicine

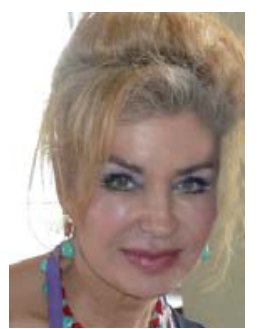

Aurora Guerra-Tapia Tenured Lecturer in Dermatology, Complutense University of Madrid; Head of the Department of Dermatology at the University Hospital 12 de Octubre, Madrid

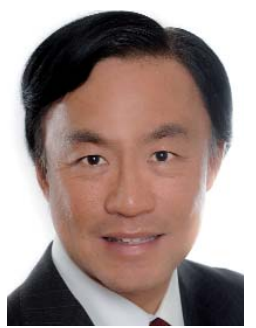

John Y. M. Koo

Professor and Vice Chairman at the University of California, San Francisco, Department of Dermatology; Director, University of California, San Francisco, Psoriasis and Skin Treatment Center

\section{EDITORIAL}

With new developments in medicine and technology, the global population is living longer than ever before. This means that the elderly population will constitute an increasing number of patients in dermatology.

The «elderly» is often defined as the adult population aged 65 and older. In 1996, it was estimated that more than $43 \%$ of the world's oldest adults lived in four countries: Japan, India, the People's Republic of China, and the United States ${ }^{1}$. One out of five Europeans are sixty or older ${ }^{1}$. It is estimated that, by the year 2050, one-fifth of the world's population will be 65 years or older ${ }^{2}$.

There are many challenges facing dermatologists when managing the care of elderly patients. First, the number of patients with skin conditions increases with age. The skin of elderly patients is both functionally and structurally different from that of younger age groups. The epidermis is thinner and has slower cell turnover, thereby, increasing vulnerability to trauma and poor wound repair. In the elderly, the sebaceous and eccrine glands have reduced functionality, resulting in xerosis and pruritus. Furthermore, there are other cumulative effects that contribute to dermatological problems in the elderly, including: UV exposure of the skin; decreased vascular flow; sweating; subcutaneous fat; slower growth of nails; dermal thinning; and a reduction of fibroblasts, mast cells, elastin, etc. ${ }^{3}$. In addition to xerosis, increased pruritus occurs in the elderly, due to neurodegenerative changes, a diminished epidermal barrier, and a reduced capacity for barrier repair ${ }^{4}$. It is known that, as a result of these changes in the skin, a number of chronic dermatologic conditions become more frequent in the elderly population, 
Wong JW et al. Introducing «dermatogeriatrics»: the need for geriatric dermatology as a subspecialty

including psoriasis, nummular eczema, xerotic eczema, bullous pemphigoid, decubitus ulcers, purpura, and chronic venous insufficiency.

Second, it is important for dermatologists to remember that with older age, patients experience age-related immunosuppression, making them more vulnerable to infection and cancer. For patients with chronic, debilitating illnesses, such as psoriasis and psoriatic arthritis, the use of immunosuppressants is a concern because of the already relatively immunosuppressed status of the elderly. Long-term immunosuppressive agents include biologic therapies, such as etanercept, adalimumab, ustekinumab, and infliximab, as well as non-biologic options such as methotrexate and cyclosporine. Moreover, there are many more immunosuppressive agents for psoriasis that are undergoing clinical trials. In addition to immunosuppression, other therapies may also be toxic to organ systems, including the cardiovascular, renal, neurological, and hepatic systems. Indeed, the presence of comorbidities is also a major concern for the elderly. Comorbidities include, among many others, metabolic syndrome, cancer, hypertension, dementia, renal failure, diabetes mellitus, and poor hepatic function, such as cirrhosis.

Third, there are also a variety of significant psychological and environmental concerns specific to the elderly. Elderly patients often have decreased mobility. For example, simply from a medical point of view, phototherapy is a systemically safer method for treatment of elderly patients with psoriasis and eczema. However, being able to stand in the boxes is a requirement for phototherapy, which is frequently challenging for the elderly. Moreover, some elderly patients are not even able to transport themselves to the clinic for phototherapy, because of arthritis, hip impairment, or a lack of transportation. Taking public transportation can even be a challenge, due to limited stamina or dementia. In addition, depression, anxiety, and isolation can often lead to the immobilization of elderly patients ${ }^{5}$.

Currently, there is no cohesive discipline within dermatology that brings together the numerous relevant issues unique to the elderly population. Consequently, these different immunological, logistical, and psychological issues of the elderly are being handled in a disjointed way by different practitioners of dermatology. As a result, there is very little activity in facilitating a clinical discussion to reach a consensus for optimal elderly care. Moreover, there is virtually no research in dermatology that specifically targets adults over the age of 65 . Hence, there is very little known about the effects of systemic therapies on the geriatric population. We cannot afford to continue having these critical issues handled in such a fragmented manner for this important age group. A new subspecialty in dermatology is needed to optimize the safety and efficacy of the clinical management of treatment and care for the geriatric population.

The establishment of geriatric dermatology, «dermatogeriatrics», as a subspecialty is essential for dermatologists, so that they have a strong foundation in understanding and treating the population who, in the near future, will constitute an increasing proportion of their patients. We propose that the mission for dermatogeriatrics would be to optimize care for geriatric patients, by understanding the physiology and psychology of this population, by increasing research, and by facilitating the clinical discussion to formulate optimal guidelines and to reach a consensus about the dermatological care of the elderly.

\section{REFERENCES}

1. Norman RA. The demographic imperative. In: Norman RA, ed. Diagnosis of aging skin diseases. London: Springer; 2008. p. 1-4.

2. Jackson SA. The epidemiology of aging. In: Hazzard WR, Blass JP, Ettinger WH Jr, Halter JB, Ouslander JG, eds. Principles of Geriatric Medicine and Gerontology, 4th ed. New York: McGraw-Hill; 1999. p. 203-25.

3. Laube S. Skin infections and aging. Ageing Res Rev. 2004;3: 69-89.

4. Berger TG, Steinhoff M. Pruritus in elderly patients-eruptions of senescence. Semin Cutan Med Surg. 2011;30:113-7.

5. Sampogna F, Chren MM, Melchi CF, Pasquini P, Tabolli S, Abeni D, and The Italian Multipurpose Psoriasis Research on Vital Experiences (Improve) Study Group. Age, gender, quality of life and psychological distress in patients hospitalized with psoriasis. Br J Dermatol. 2006;154:325-31. 